DELPIERRE Pierre /EP

DELPIII 87-78 CAI 48

25 September 1987

\begin{abstract}
ALIGNMENT IN MAGNETIC FIELD, CHARGE
TRANSMISSION AND DRIFT VELOCITY MONITORING

FOR THE TPC-IIKE ELECTROMAGNETIC CALORIMETER

OF THE DEIPHI EXPERIMENT AT IEP
\end{abstract}

M. Sannino and E. Durante ${ }^{*}$

*) Not a member of DEL.PHI 


\title{
Alignement in Magnetic Field, Charge Transmission and Drift Velocity Monitoring for the TPC-like Electromagnetic Calorimeter of Delphi Experiment at LEP
}

\author{
M.Sannino ${ }^{(1,2)}$ and E.Durante ${ }^{(1)}$
}

(1) INFN sez. di Genova, Genoa, Italy

(2) Dipar timento di Fisica, University of Genova, Genoa, Italy

\begin{abstract}
:
The HPC, electramagnetic calorimeter for the LEP Delphi experiment, is a TPC-like detector warking in B field. Its response deperids much on charge transmission to reading plane, which requiress a good alignment between $\mathbf{E}_{d}$ and $\mathbf{B}$ fields, and on drift velocity. To align $\mathbf{E}_{d}$ and $\mathbf{B}$ fields and to monitor charge transmission, the signal of $\alpha$ particles, emitted by a source in a drift channel near HT-plane, will be measured. Some HPC modules will be equipped with a $\mathrm{P}-\mathrm{I}-\mathrm{N}$ photodiode, faced to the source, in order to trigger $\alpha$ particles so to monitor drift velocity. Tests in a drift channel simulating HPC were performed and gave good results for this monitoring method also for what drift velocity is concerned.
\end{abstract}

Preserited at the London Conference on Position Sensitive Detectors $7^{\text {th }}-11^{\text {th }}$ September 1987, London, UK 


\section{Introduction}

Recently a new TPC-like calorimeter, the High Density Projection Chamber (HPC), has been proposed for Deiptri-LEF detector and is now teing built $(1,2,3)$. Each module of this calor imeter (fig. 1) is in fact, made up by many narrow and long drift channels separated by lead carverters and works as follows. A primary photon or an electron generates a shower in the drift channel structure, made up of lead wires acting as converters and drift field shaping electrodes, and ionizes the grs ( $80 \% \mathrm{Ar} \div 20 \% \mathrm{CH}_{4}$ mixture). The ionization electrons drift along the channels under the action of a uniform electric field $\mathbf{E}_{d}$ and finally enter a proportional chamber with pad reaj-out. Each pad is faced to a number of drift channels between 3 and a multiple of 3 or 4 and a multiple of 4 , deperiding on the pad. Appropriate encoding elactronics gives then a three dimensional view of the shower profile together with the energy deposited by the showering particle. Moreover this calor imeter has to work inside a solenoidal magnetic field $\mathbf{B}$ parallel to the electric arift field $\mathbf{E}_{\mathrm{d}}$. The response of such a type of calorimeter to given energy releases essentially depends on:

i) Lorization charge transmission to the reading plane, which in turn depends on:

a) Good alignment between $\mathbf{E}_{d}$ and $\mathbf{B}$ fields.

b) $\mathbf{E}_{d}$ uniformity and gess pur ity (e.g. abserice of electronegative polluants) in drift structure.

i1) Uniformity of reading pads response, that means uniformity of gain of proportional tubes of which pads are made.

The spatial resalution of the detector depends on:

iii) Drift velocity of ionization electrons in gas.

Quantities related to points i), ii) and iii) above must be monitored in order to know the response of the calorimeter at every time during data taking.

More in detail these quantities may be chosen as:

1) Pulse height response of a given pad to a fixed quantity of energy released by ionization in a position next to HT side of a corresponding drift channel (fig. 1). This energy release should be of the same order of magnitude of the energy 
released by an electromagnetic shower. In this way ionization charge transmission to reading plane is monitored and it is possible to get a calibration point.

Charge tranismission monitoring is a good method to align $\mathbf{E}_{d}$ and $\mathbf{B}$ fields, ass it will be discussed later.

2) Drift time of ionization electrons, generated by particles used to obtain the energy release mentioned in point 1) above, allowing so a measurement of drift velocity of electrons in gas.

3) A periadical check of the uniformity of response of all pads to m.i.p. traversing or thogonally the drift structure (e.g. cosmic rays). This permits equalization of pads at run-time. Of course this procedure, which exploits cosmic rays, is very long and may be done from time to time in between data-taking runs.

Exploiting ideas exposed in points 1) and 2) above, we developed a system which allows on-line monitoring of ionization charge transmission and electrons drift velocity.

\section{Implementation of the method using an alpha source}

The enerov released in the gas filling a HPC drift channel by a typical shower is of the order of some huridreds $\mathrm{KeV}$ as from MC simulations. So, as every pad is faced to a minimum number of 3 arift channels (see above) - depending on position along the lorigitudinal development of the shower - a good enough energy release in the gap, to get a calibration point, might be between 100 and $400 \mathrm{KeV}$.

Such an energy release may be obtained using $\alpha$ particles emitted perpendicularly to drift direction by a collimated radioactive source; source is located near HT side of a HPC drift channel (fig. 1). Normally a particles emitted by sources have energies of the order of $5 \div 6 \mathrm{MeV}$ (e.g. main line of ${ }^{241} \mathrm{Am}$ is at $5.48 \mathrm{MeV}$ ): $\alpha$ particles of such energies loose by ionization, in a gas mixture at S.T.P.(Standard Temperature and Pressure), an energy of the order of $100 \mathrm{KeV}$ for each millimeter of gas traversed (4). This means a moximum of $800 \mathrm{KeV}$ of energy released if collimated $\alpha$ particles are emitted in a direction perpendicular to the lead planes defining the drift channels and are allowed to ionize for the full height of the drift channel $(8 \mathrm{~mm})$ without absorbing them; smaller energy releases are possible by means of an absorber in front of the source.

Therefore, monitoring dur ing the data taking the pulse height response of the pad faced 
to a particle ionization, it is possible to know the evolution in time of the response of the calorimeter, concerning charge transmission in the drift region provided gas proportional gain is moritored. This allows to obtain a calibration of the HPC.

\subsection{Alignment in magnetic field}

The respornse of the pad to $\alpha$ particle ionization depends on charge transmission inside the dr ift channel which, in turn, is strongly dependent on the alignment between $\mathbf{E}_{d}$ and B fields, and is maximum when the two fields are parallel to each other. The measurement of this response is, so, a good methad to align the module in the magnetic field.

This alignment procedure relies upan the results of a previous work ${ }^{(5)}$ performed by one of the authors: the aim of such a work was to measure attenuation lengths of electrons drifting in $80 \mathrm{PAr} \div 20 \mathrm{OCH}_{4}$ in a narrow $(\approx 8 \mathrm{~mm}$ height $)$ and long $(\approx 1 \mathrm{~m})$ drift chamel when a solenoidal magnetic field, parallel to the drift electric field, is present. The drift channel was built in such a way as to simulate a HPC drift channel and it was similar, but longer, to the one described below in paragraph 3.2. In this long drift channel a collimated ${ }^{241}$ Am source, emitting $\alpha$ particles, was located near the HT side of the drift channel and the response of the proportional tube, as a function of the relative or ientation between $\mathbf{E}_{d}$ and $\mathbf{B}$ fields, was measured. Two angular scans were made, one in the vertical plane (angle $\phi$ ) and one in the horizontal plane (angle $\theta$ ) in order to display respectively the so called ( $\mathbf{E} \bullet \mathbf{B}$ ) B effect and EXB effect; results are reported in fig. 2; see fig. 5 for reference frame.

From these data it is evident that the signal of the proportional tube, due to $\alpha$ particles, is maximum when $\mathbf{E}_{d}$ and $\mathbf{B}$ fields are aligned. As a conclusion, this procedure may be exploited to align HPC modules in B field when an ${ }^{241} A m \alpha$ source is installed on the HT side of a HPC drift channel.

\subsection{Drift velocity monitoring}

It is important to monitor, in some HPC modules, drift velocity of ionization electrons in HPC drift channels under real operating conditions. A real-time knowledge of drift velucity is, infact, fundamental for a reliable spatial resolution of the detector. To measure orift velocity a "zero time "is needed and this may be obtained by detecting the 
collimated $\alpha$ particles, used to monitor the HPC module, after they have traver sed the gas gap. Alpha particle detection may be accomplished by means of a $P-1-N$ photodiode settled in front of the $\alpha$ source (fig. 1). The signal of the diode is used to give the start to a clack which is stopped by the signal of the pad faced to the concerned dr ift chiannel.

\section{Feasibility tests of the drift velocity monitoring system}

\section{I Preliminary tests on $P-1-N$ photodiode}

As a preliminary test for the drift velocity monitoring system, we exposed to an ${ }^{241_{A m}}$ $\alpha$ source (Main line at $5.48 \mathrm{MeV}$ ) a $P-1-N$ photadiade type SIEMENS BPX 61 (dimensions $3 \times 3 \mathrm{~mm}^{2}$ ), chosen for its low reverse current $(\approx 1.5 \mathrm{nA}$ ) and its low capacitance $(\approx 10 \mathrm{pF})$ under a reverse polarization of $27 \mathrm{~V} ; \mathrm{P}-1-\mathrm{N}$ phatodiodes of this type were alreaty used as a source spectrum detector (6). The P-I-N photodiode preamplifier, developed by J.C.Santiard at CERN ${ }^{(8)}$, is a fast (risetime 20 ns) charge sensitive type with FET input.

Spectra of diode signal, which, after preamplification, has a risetime of 20 ns, have bean mesasured in vacuum (iig. 3) and in air at S.T.P. (fig. 4) for a distance between diade and source of $s: 8 \mathrm{~mm}$. The spectrum of $\alpha$ particles in air (fig. 4) shows a displacement of peak toward lower values, due to energy lost by $\boldsymbol{\alpha}$ particles in collisions with air molecules. The difference between positions of peaks in figs. 3 and 4 allows to measure the energy released by $\alpha$ particles in $8 \mathrm{~mm}$ of air, provided the linear ity of the diode reading chain is checked.

The result is

$$
E_{\text {rel }}=0.82 \pm 0.14 \mathrm{MeV}
$$

This medsurement is in a good agreement with what foreseen by Range-Energy tables for 5.48 MeV $\alpha$ particles from ${ }^{241}$ Am that traverse $8 \mathrm{~mm}$ of air $(4,7)$ that is

$$
E_{\text {rel }}=0.76 \mathrm{MeV}
$$

From this test we obtained confidence that the $\mathrm{P}-1-\mathrm{N}$ photodiode has a good performance as a fast and chead $\alpha$ particle detector and is a good candidate to trigger $\alpha$ particles.

\subsection{Installation of a $\mathrm{P}-\mathrm{I}-\mathrm{N}$ photodiode in a drift channel}

To test the feasibility of $\alpha$ particle triggering system by means of a $\mathrm{P}-1-\mathrm{N}$ photodiode in 
a stuation pretty analogous to the real one we have installed an $\alpha$ source, with a $P-i-N$ photodiode in front, on the HT side of a drift channel simulating a HPC drift channel ( see fig. 5)

The single drift channel of HPC was built using lead wire ribbon, i.e the same tectmique (3) chosen for the construction of the fina? calorimeter. Seventeen ribbons, each made up of 20 parallel lead wires, 1.7 mm thick, separated by 0.2 mm and glued on both sides of a thin vetronite support, were bent to form $U$-shaped pieces which were alignad all tugether on a plexiglass support in order to constitute a single drift channel. Resistors were placed between each contigous lead wires to let them act as field shiaping electrodes. The size of the drift channel is $616 \times 100 \times 8(X \times Y \times Z) \mathrm{mm}^{3}$. The $\alpha$ source is installed below the lead wires and the $\alpha$ particles are injected into the channel through a $81 \mathrm{~mm}$ hole at $610 \mathrm{~mm}$ from reading proportional tube. The $P-1-N$ photodiode, of the same type as in prevtous paragraph, is inside the drift channel in front of the hole injecting $\alpha$ particles in the drift channel. The path of $\alpha$ particles in gas inside the drift channel is $\approx 6 \mathrm{~mm}$ before being absorbed and detected by the $P-1-N$ photodiode so that they loose about $600 \mathrm{key}$ in gas. The ionization signal is read by a proportional tube of the same type as the ones making up the pads of the HPC reading chamber: in order to accomplish this test the signal is taken out of the proportional wire and is fed into a standar L LABEN charge preamplifier. The $P-I-N$ photodiode is inserted in a floating circuit in series with a $10 \mathrm{M} \Omega$ resistor and with a polarizing battery insulated from the ground. This is dorie as the P - I-N photodiode is settled in the HiT side of the drift channel so it sets at high tension when drift voltage is switched on. The $P-1-N$ photodiode signal is therefore fed into the preamplifier through a $H T$ capacitor as may be seen from the scheme (fig. 6). The $\mathrm{F}-1-\mathrm{N}$ photodiode preamplifier is of the same type mentioned in previous paragraph.

\subsection{Operation of the system - Experimental results}

As in the real situation the system was operated in a $80 \% \mathrm{Ar} \div 20 \% \mathrm{CH}_{4}$ gas mixture : the result was a good and fast ( $r$ isetime $20 \mathrm{~ns}$ ) signal from the $\mathrm{P}-1-\mathrm{N}$ photodiode which, in turn, allowed a stable trigger for the proportional tube signal. An evident example of this may be clearly seen in fig. 7 where the two signals are added on the scope. The drift time in fig. 7 is $14.3 \mu$ with an electric drift field $E_{0}=80 \mathrm{~V} / \mathrm{cm}$ corresponding to a drift velocity $y_{\mathrm{f}}=4.3 \mathrm{~cm} / \mu \mathrm{s}$, so in fair agreement with literature data ${ }^{(9)}$; for a measument at a different value of $\mathrm{E}_{d}$ see table 1 . The spectrum of the corresponding wire 
signal, triggered by the diade, is reported in fig. 8. The response of the proportional tube to a particles has been measured at different valtages of the sense wire; the measurements are reported in fig. 9 where it can be seen that, only for a proportional wire terssuon larger than $1300 \mathrm{~V}$, there is saturation, the gain still increasing in an exponential way for lower values of tension. As the HPC reading chamber is for eseen to be operated at $=1250$ V this makes us confident that this system may be used for data-taking calibration and manitoring despite the fact that ionization density due to $\alpha$ particles is munh higher than the one due to a shower.

Just for sake of comparison the spectrum of the wire signal due to $\alpha$ particles with no photodionde trigger is presented in fig. 10 where it may be seen that the position of the peak is always the same but the FWHM of the distribution is $\approx 40 \%$ larger.

\section{Conclusion}

We have described a method to monitor charge transmission and drift velocity in HPC drift chanineis using an $\alpha$ source and a $\mathrm{P}-1-\mathrm{N}$ photodiode facing the source. This procedure is also useful to align the drift electric field of HPC in the Delphi solenoidal

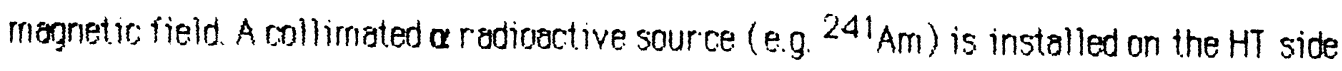
of a HPC drift channel: the ionization electrons due to a particles are collected on the pickup chamber. Therefore, measuring the corresponding pulse height in a facing pad, we get a monitor of charge transmission in the drift structure. The $\mathrm{P}-1-\mathrm{N}$ photodiode, installed in front of the source, detects $\alpha$ particles giving a "zero time" and so allowing a monitor of drift velocity in the gas under real operating conditions. Tests in a long drift channel simulating HPC gave good results.

Acknowledgments

We are particularly grateful to V.Gracco for many interesting and very useful discussions and for his constant stimulus and encouragement. One of US (MS) is grateful to G. Matthias and 0.vllaland for a fruitful discussion on the method. M.Castoldi is gratefully acknowledged for an interesting discussion on preamplifiers for solid state detectors. The gas system was a skillful work by A. Morelli. 
Table 1

Measured drift velocities for different electric urift fields

$\begin{array}{cc}\left|E_{d}\right| & v_{d} \\ {[V / \mathrm{cm}]} & {[\mathrm{cm} / \mu \mathrm{s}]}\end{array}$

80

4.3

140

5.7 


\section{REFERENCES}

1) H.G. Fischer and 0. Uilaland - IEEE Transactions Nuci. Sci. NS-27(1980)38

2) Delphi - Technical Proposal - CERN/LEPC/83-3/, LEPC/P 2, 17 maY 1983

3) Delphi - Progress report - CERN/LEFC/84-16, LEPC/PR 6, 26 september 1984

4) See range-eneroy tables e. L.C.Northcliffe and R.F.Schilling - Nuclear Data Tables A7(1370)233

5) V.Graco, L.Rosi, M. Samina, L.Traspedini-Nuci. Instr and Meth. A252(1986)573

6) J. Dousse and ch Rreme - Am.J.Prys 51 (1983)452

7) M.S.Livingston and H.A.Bethe - Rev. Mod. Phys. 9(1937)245

8) U.C. Sanitiard - CERN EP Internal Revort 85-05(1985)

9) A.Petsert and F. Sauli - Yellow Repurt CERN 84-08 
FIGURE CAFTIONS:

Fig. 1 Detail of a HPC module. The sketch of a shower is superimposed to the drawing in arder to imustrate how the calorimeter works (see text for details). An indicative sketch of the installation of the 241 Anr radioactive $\alpha$ source and $P-1-N$ photodiode is shown ton.

Fig? From ret. 5: Mean pulse amplitude due to a particles ionization generated near. HT side of a $=1 \mathrm{~m}$ long and narrow ( $8 \mathrm{~mm}$ ) drift channel read by a proportional tube at different or ientations between electric drift field and magnetic field: ( $E \bullet B$ ) B (angle 6) and $\mathrm{E} \times \mathbf{B}$ (angle $\theta$ ) effects are shown (reference frame as the one defined in fig.5), the solid lines are simulations results (for more details see ref.5).

Fig. Spectrum in vacuum of $P-1-N$ photodiode exposed to $241_{A m} \alpha$ source at a distance $\approx 8 \mathrm{~mm}$ ( see text).

Fig 4 Spestrum in air at STP of $P-1-N$ photodiode signals exposed to $241_{\text {Am } \alpha \text { source at a }}$ distance $=8 \mathrm{~mm}$ (see text).

Fig.5 Schematic view of drift channel simulating one HPC drift channel with $\alpha$ source and $P-I-N$ photodiode near HT side; the reference frame, valid also for fig 2 , is shown.

Fig. 6 Scheme of the $P-1-N$ photadiode reading circuit ( see text).

Fig. $7 \quad P-1-N$ photodicde trigger signal due to $\alpha$ particles and triggered proportional wire signal seen after drift time added on the scope ( $\left.H V_{\text {wire }}=1250 \mathrm{~V}\right)$. Hor. scale $2 \mu \mathrm{s} / \mathrm{div}$, Vert. scale $10 \mathrm{~m} V /$ div for both signals (see text).

Fig.s Snectrum of proportional wire signal triggered by $\alpha$ particles signal an $\mathrm{P}-\mathrm{I}-\mathrm{N}$ photodiode for $\mathrm{HV}_{\text {wire }}=1250 \mathrm{~V}$.

Fig.9 Mean pulse height of proportional wire signal due to $\alpha$ particles vs. proportional wire voltage.

Fig. 10 Spectrum of proportional wire signal due to $\alpha$ particles when no $\mathrm{P}-1-\mathrm{N}$ photodiode trigger signal is present for $\mathrm{HV}_{\text {wire }}=1250 \mathrm{~V}$. 


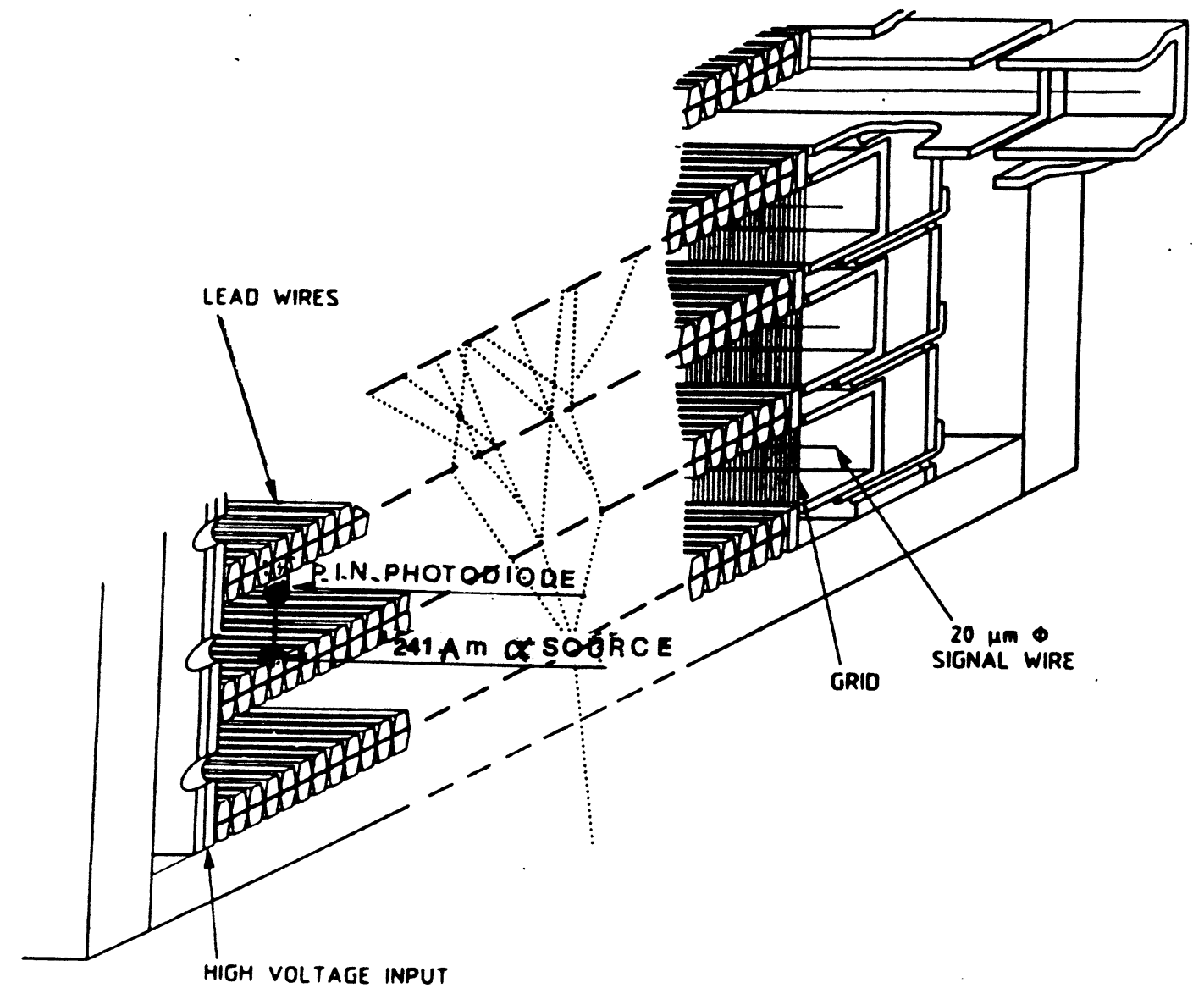

Fig.1 


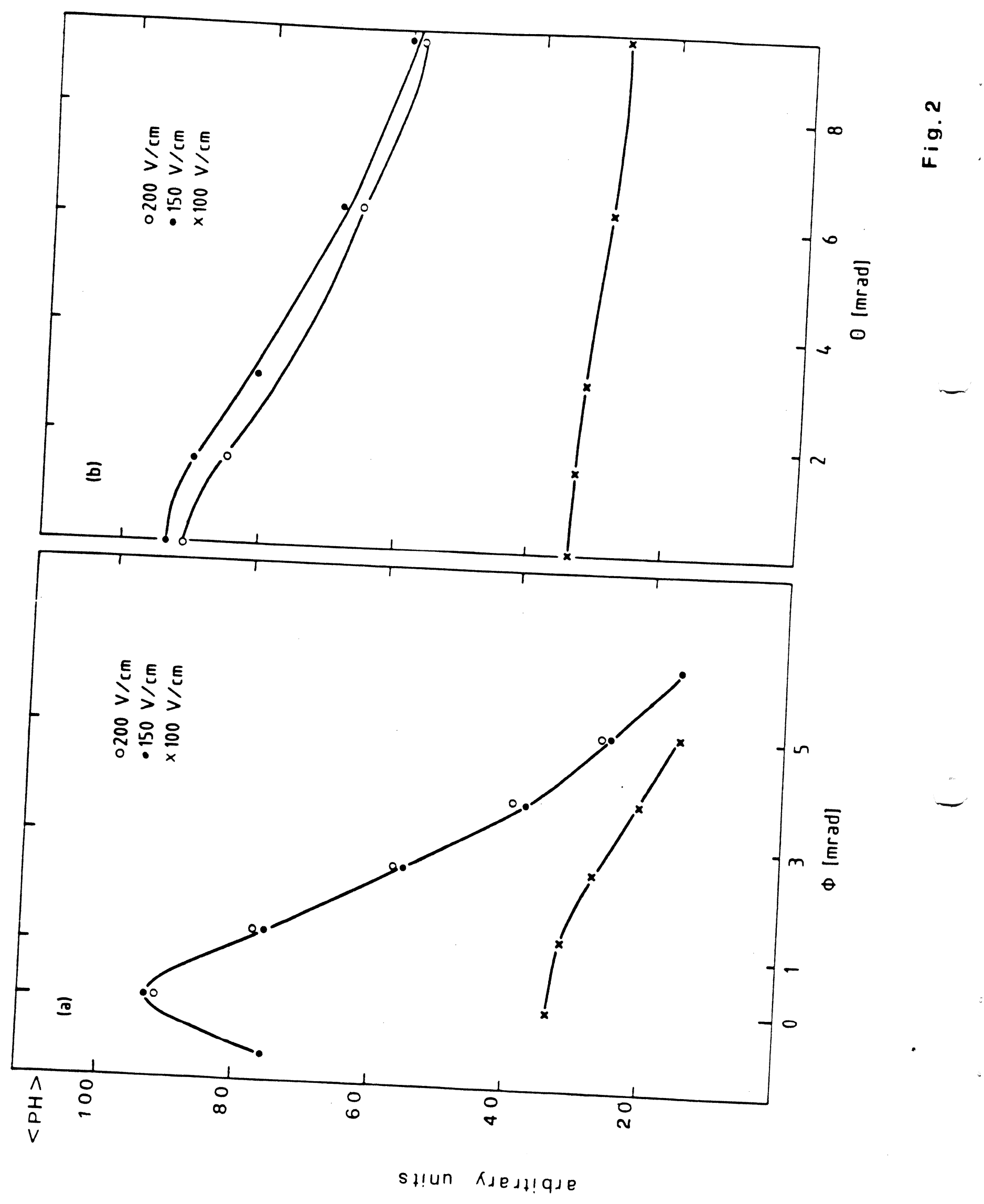




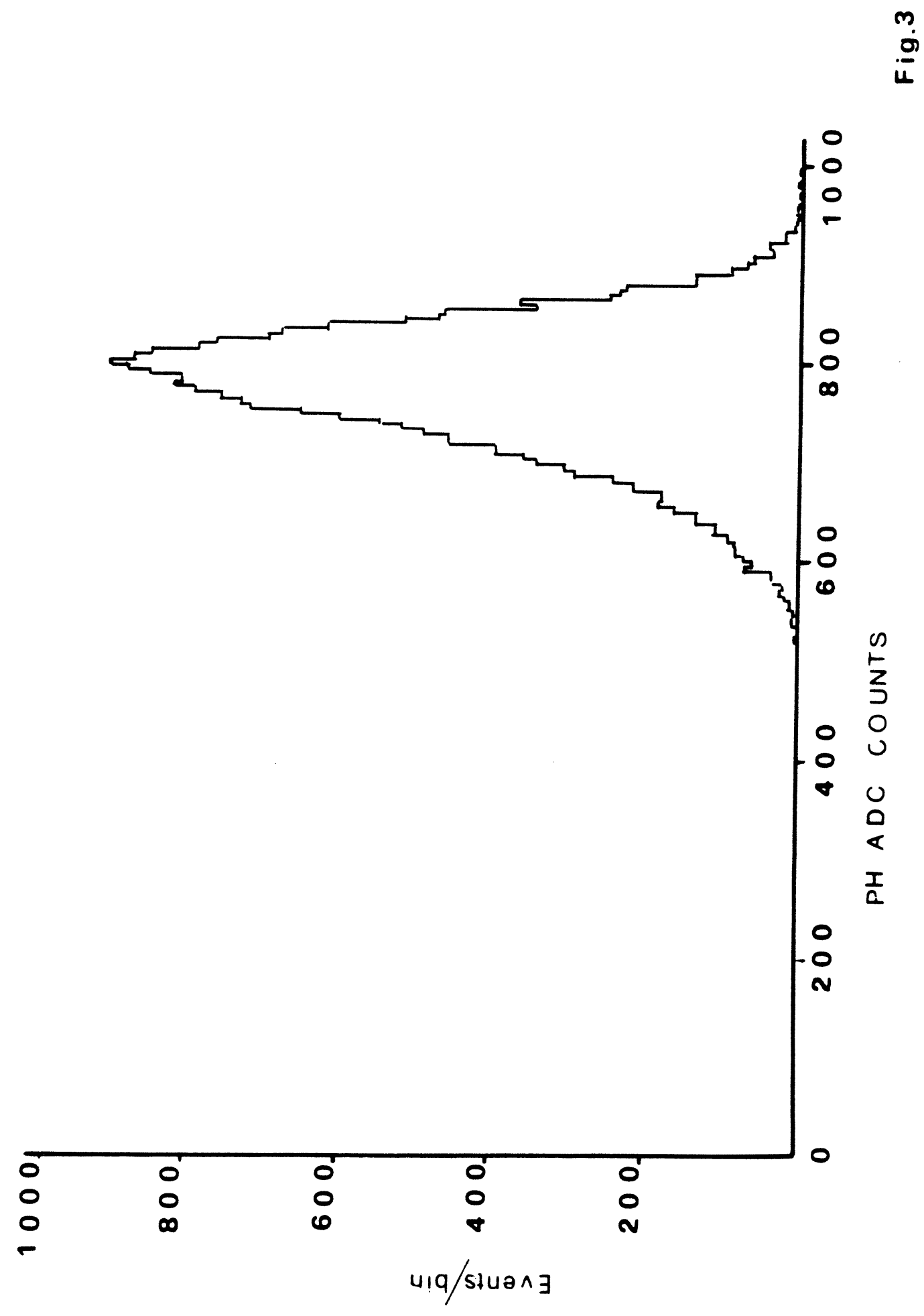




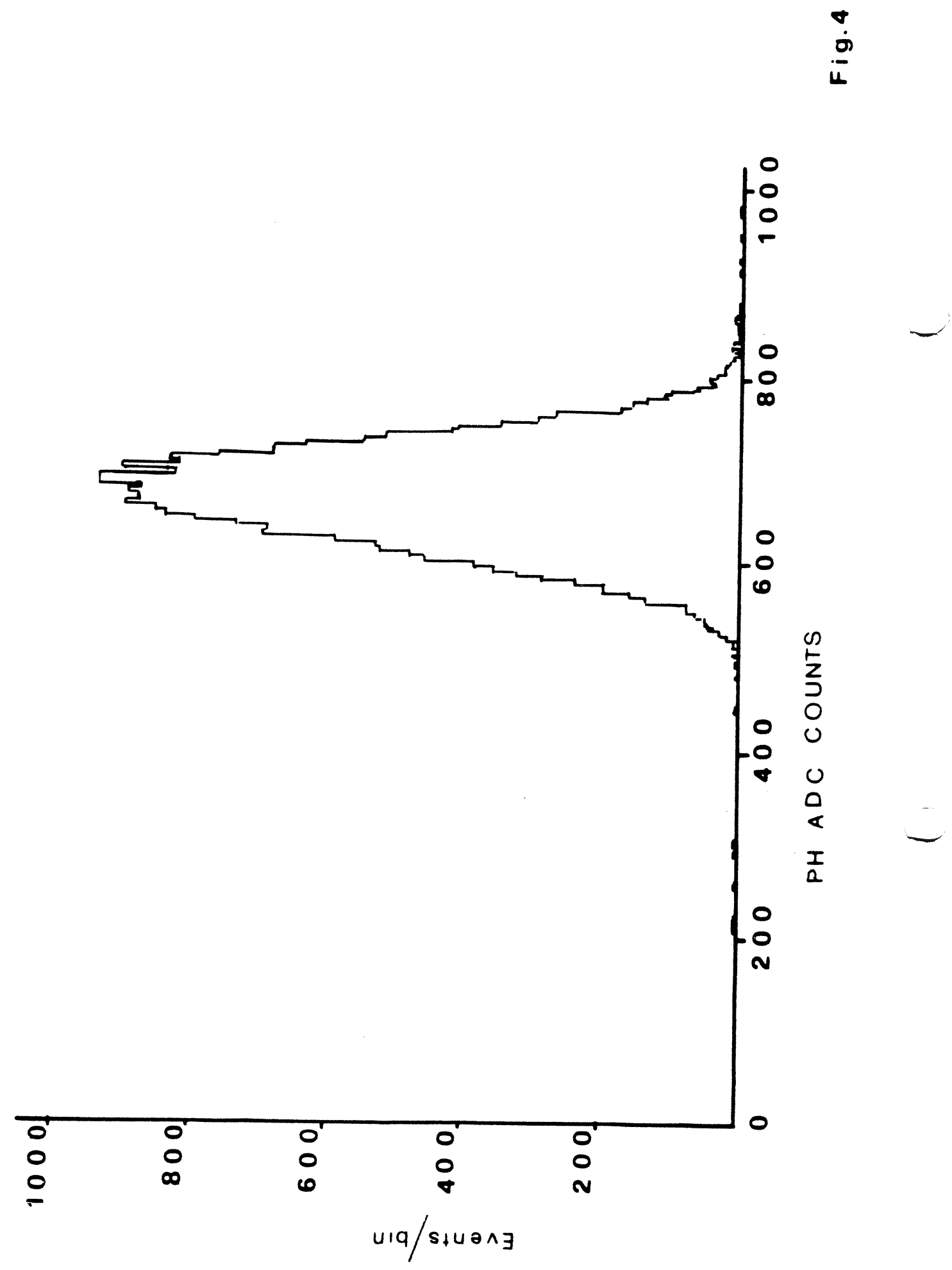




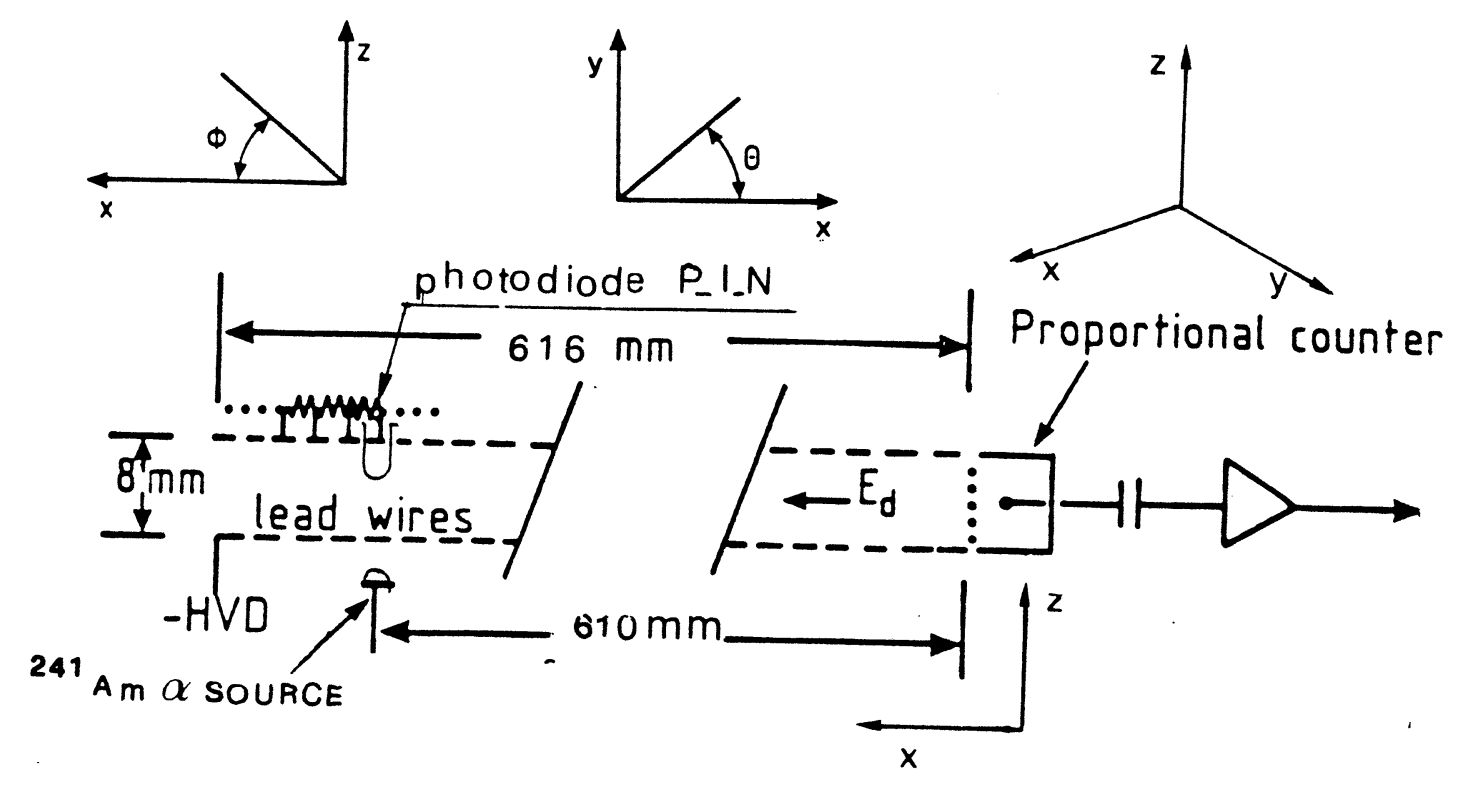

F ig. 5 


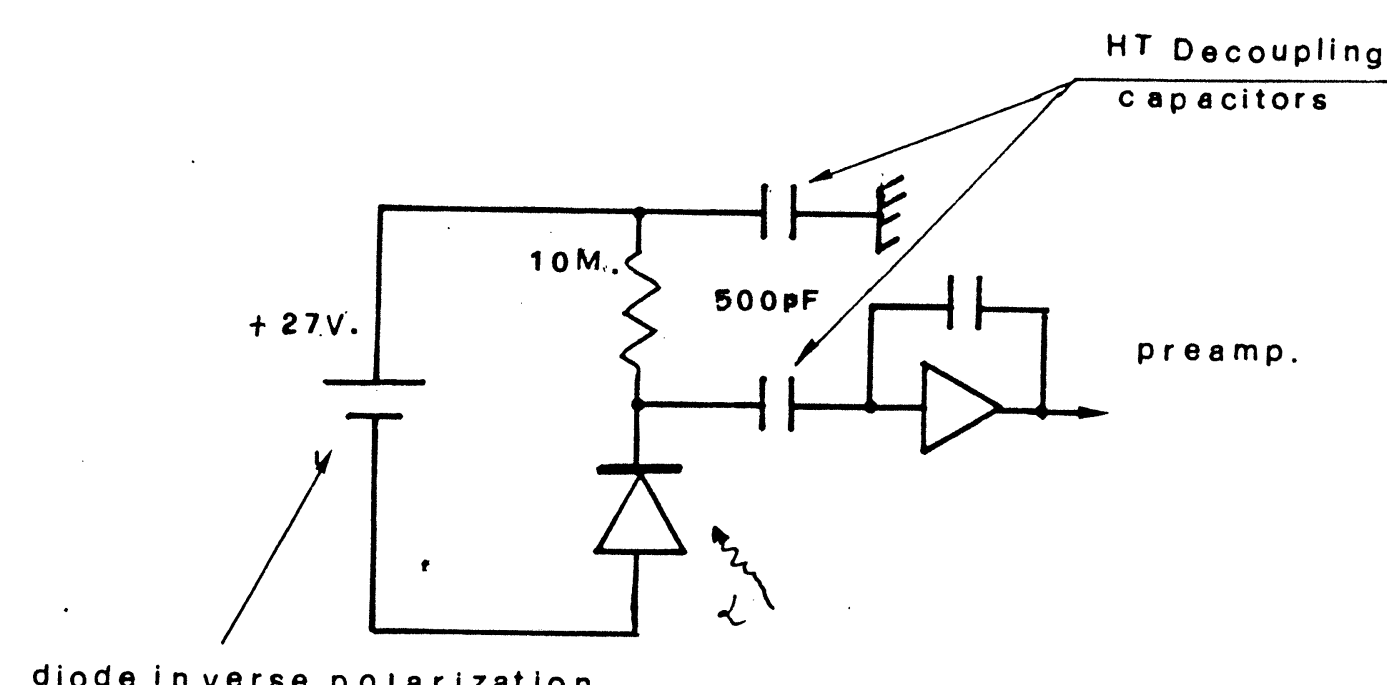

Fig. 6 


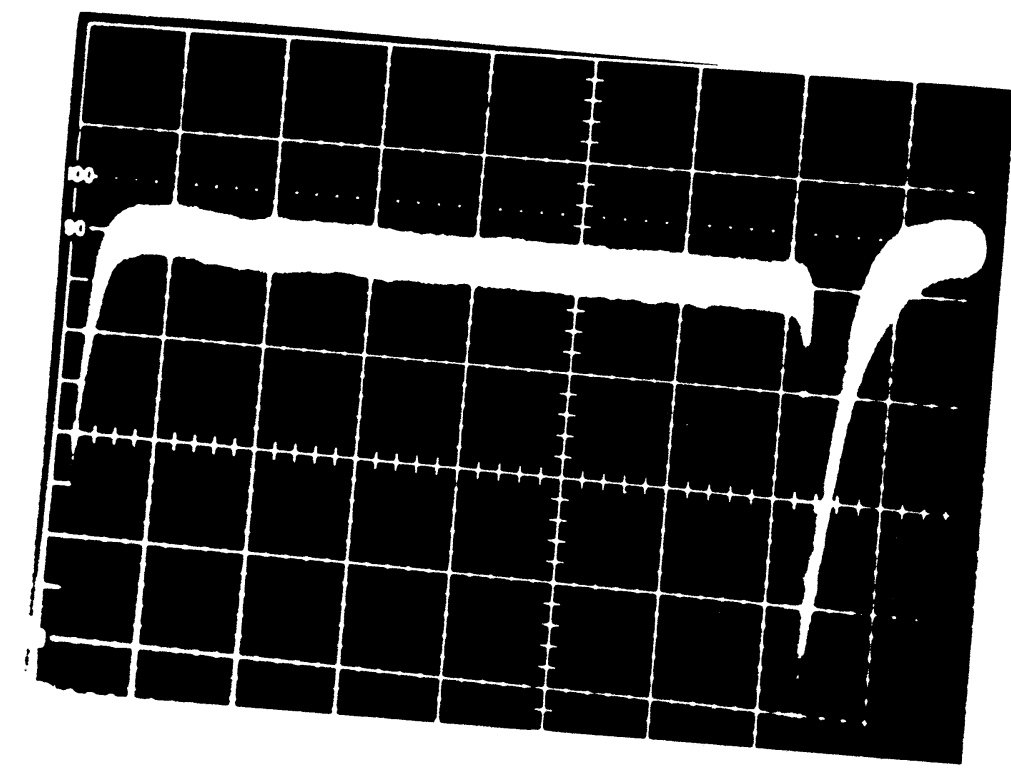

Fig. 7 


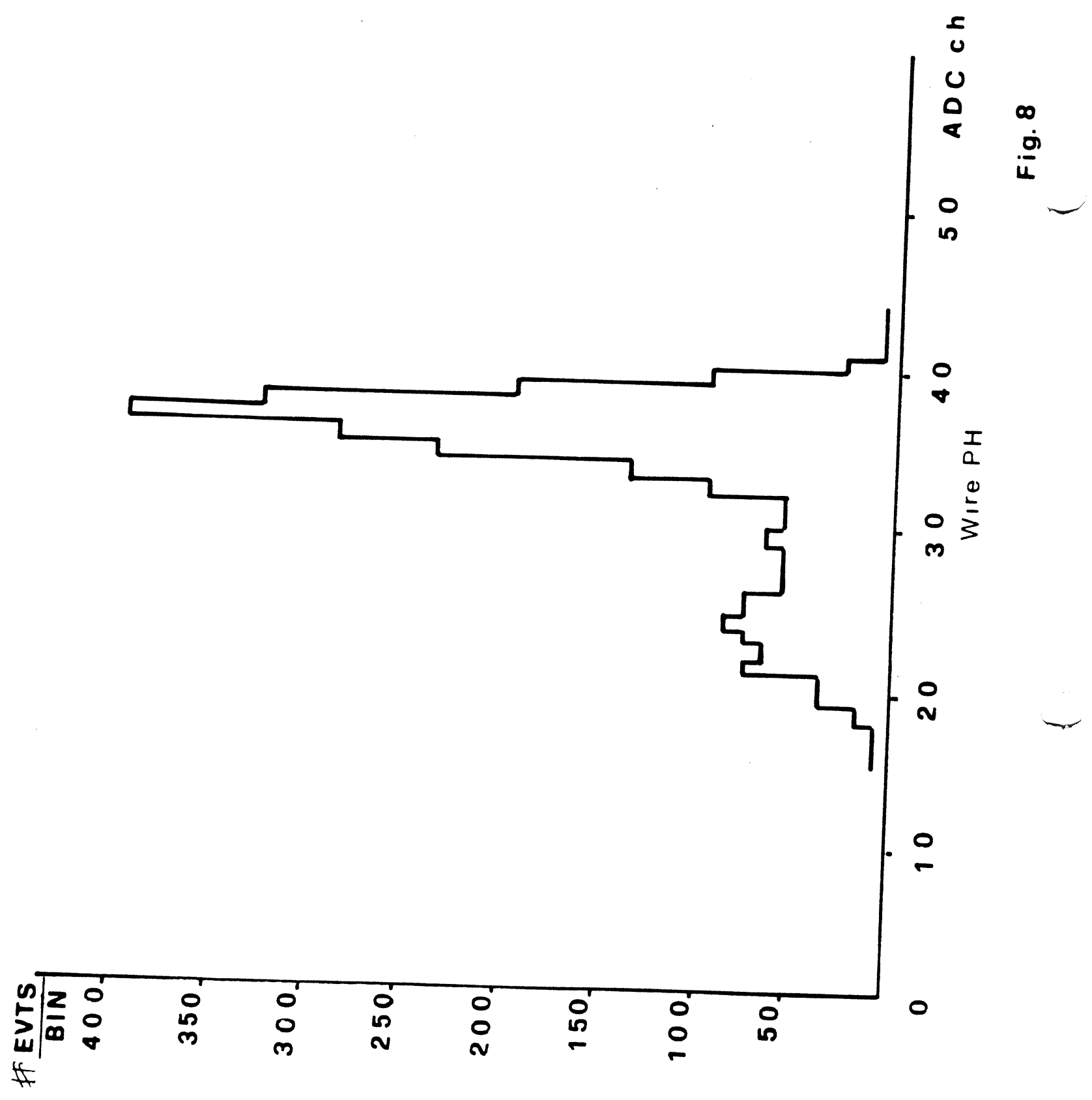




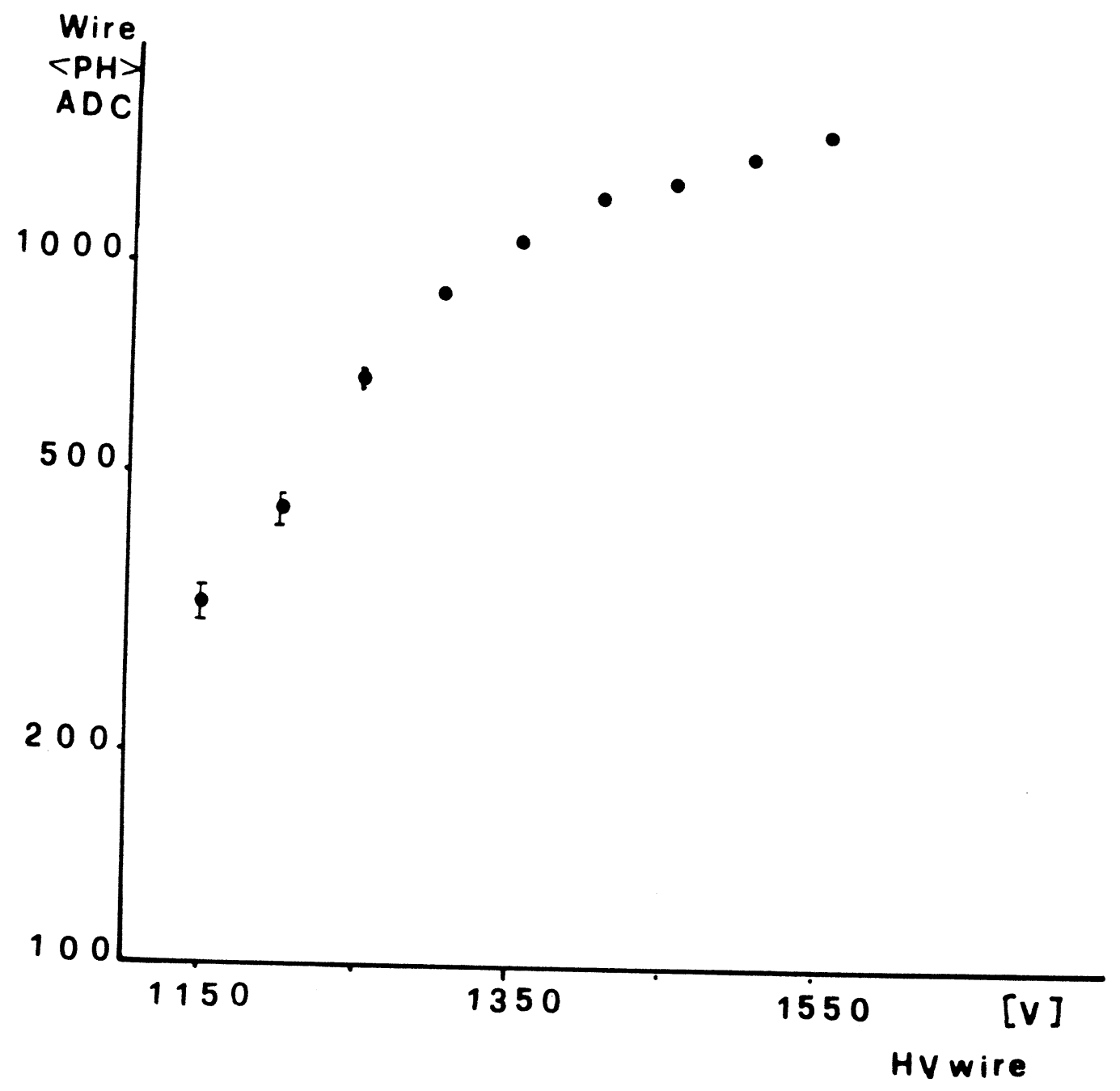

Fig. 9 


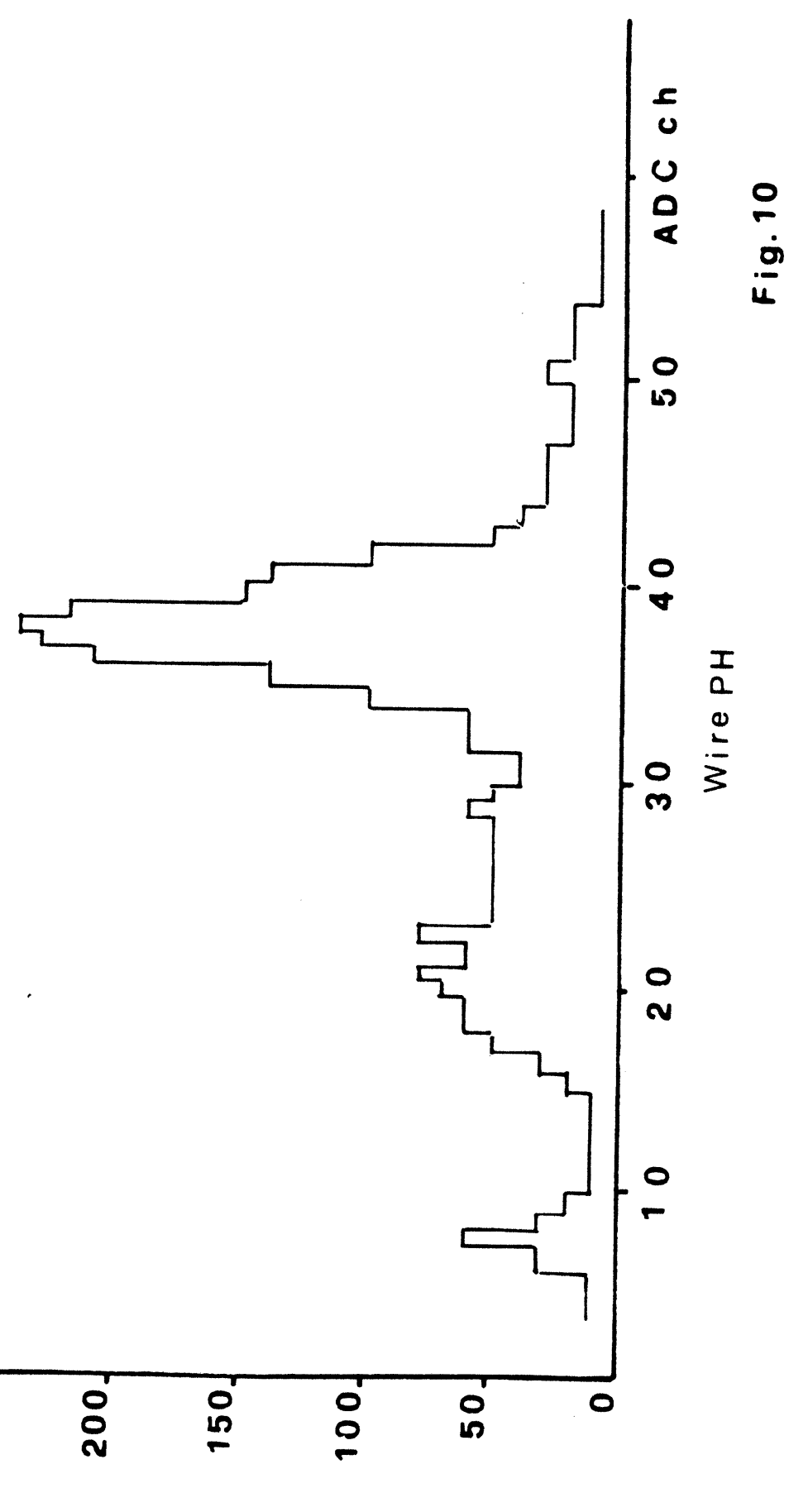

